\title{
Implementation of discrete positive operator valued measures on linear optical systems using cosine-sine decomposition
}

\author{
Jaskaran Singh $\odot,{ }^{*}$ Arvind, ${ }^{\dagger}$ and Sandeep K. Goyal $\odot^{\ddagger}$ \\ Department of Physical Sciences, Indian Institute of Science Education \& Research (IISER) Mohali, \\ Sector 81 SAS Nagar, Manauli PO 140306, Punjab, India
}

(Received 18 June 2021; revised 16 September 2021; accepted 26 November 2021; published 3 January 2022)

\begin{abstract}
Positive operator valued measurements (POVMs) play an important role in efficient quantum communication and computation. While optical systems are one of the strongest candidates for long distance quantum communication and information processing, efficient methods to implement POVMs in these systems are scarce. Here we propose an all-optical scheme to implement an arbitrary POVM using linear optical components on $m$-dimensional Hilbert space of internal degrees of freedom. Linear optical nature of the proposed scheme makes it efficient and robust. We show how the scheme can be applied for state tomography and for preparing arbitrary mixed states.
\end{abstract}

DOI: 10.1103/PhysRevResearch.4.013007

\section{INTRODUCTION}

Projective measurements play an important role in information theoretic applications of quantum theory. However, they are not the most general type of measurements or even the optimal ones in most cases [1,2]. For example, in quantum optics, the homodyne and heterodyne measurements [3,4] routinely implemented in the laboratory cannot be modeled as projective measurements. The most general class of measurements are mathematically represented as quantum instruments [5-8] with positive operator valued measures (POVMs) [1,9] being a special case of them. POVMs and their applications have been theoretically studied [10-14]; however, their experimental implemention on optical systems still remains a challenge. In this paper we propose a scheme to implement an arbitrary POVM on optical systems. The scheme uses the Naimark dilation theorem and cosine-sine (CS) decomposition [15] in order to realize the POVMs.

POVMs have proven to be advantageous in quantum state discrimination $[2,16,17]$, quantum metrology [18], quantum state and process tomography $[19,20]$, coherent controls [21], local filtering operations [22], and more recently in quantum key distribution protocols $[23,24]$. They are also significant in exploring foundational aspects including Bell nonlocality $[25,26]$ and quantum contextuality [27] where it is not clear how these scenarios hold if POVMs are considered instead of projective measurements. Specifically, in the field of quantum

\footnotetext{
*jsinghiiser@gmail.com

†arvind@iisermohali.ac.in

\$skgoyal@iisermohali.ac.in
}

Published by the American Physical Society under the terms of the Creative Commons Attribution 4.0 International license. Further distribution of this work must maintain attribution to the author(s) and the published article's title, journal citation, and DOI. contextuality, where experimental demonstrations have only been performed for projective measurements, while experimental signatures of the same for POVMs is still an ongoing research [28]. An efficient technique to perform arbitrary POVMs would greatly benefit these fields. Photonic systems are becoming an important platform for quantum information, communication, and computation. Therefore, it is of tantamount importance to have efficient schemes to realize POVMs on these systems.

In general, POVMs can be implemented on a quantum system by coupling the system with an ancillary system and performing projective measurements on the combined system. POVMs have been experimentally realized on optical systems for quantum state discrimination [29,30], quantum state estimation [31], and entanglement distillation [32]. However, these schemes require the use of a large number of beam splitters (BSs), wave plates (WPs), and other optical elements. It is also not known whether they are optimal or not. Several theoretical protocols have also been put forward for their optical implementation using polarization degrees of freedom of single- and two-photon [33-35] quantum walks [36] and also on circuit based quantum computers [37]. There also exist theoretical protocols to simulate arbitrary POVMs on a $d$-dimensional quantum system using classical randomness and postselection [38,39]. However, an efficient experimental implementation of the same has not yet been done. Further, these theoretical protocols only succeed probabilistically, with lower success probability for higher dimensions. Techniques to implement arbitrary POVMs deterministically for any dimension of Hilbert space on optical systems is unknown. This limits their applicability in quantum information and communication tasks.

In this paper we propose an efficient, scalable, and an all-optical scheme to implement an arbitrary POVM on optical systems using unitary operations and projective measurements. We use the Naimark dilation theorem and CS decomposition to realize the POVMs. Our scheme requires 
only 2(n-1) number of balanced BS and other simple linear optical elements to realize an $n$-outcome POVM. Since all the components required are linear, this makes our scheme deterministic and efficient. We explicitly provide simple optical circuits for the implementation of two-outcome POVMs and symmetric informationally complete POVMs (SIC-POVMs) on a single qubit and provide a method for realizing an arbitrary mixed state of a photonic quantum system which can be readily implemented in a laboratory with the current technology.

This paper is organized as follows: In Sec. II we review the concept of POVMs and the CS decomposition [15]. We also discuss in detail the optical setup and the allowed operations on the internal and external degrees of freedom of light. In Sec. III we present our scheme to implement $n$-outcome POVMs and the applications of the scheme are presented in Sec. IV. We conclude in Sec. V.

\section{BACKGROUND}

In this section, we present the relevant background required for understanding the results in the paper. We start with discussing the optical systems considered in this paper. Here we discuss the external and internal degrees of freedom of light and the allowed unitary operations on them. We also review the concepts of POVMs and the CS decomposition.

\section{A. Optical systems}

In this paper we primarily focus on implementing POVMs on an optical system using external and internal modes of light. Throughout the paper, the spatial modes of light are regarded as an external degree of freedom (DoF), while polarization and orbital angular momentum (OAM) modes as internal. We present a scheme to implement an $n$-outcome POVM on the internal DoF using spatial modes as ancilla systems. Although the current scheme works for any internal DoFs with an arbitrary Hilbert space dimension, we assume that one is capable of experimentally implementing unitary operations on any internal DoF of choice. This is apparent in the OAM DoF where it is still a challenge to experimentally implement arbitrary local unitaries on them.

Any arbitrary unitary operation $U(N)$ on $N$-spatial modes can be applied using the prescription given by Reck et al. [40]. In this prescription, $U(N)$ is decomposed using $O\left(N^{2}\right)$ balanced beam splitters (BBSs) and phase plates. The action of a BBS on two spatial modes is represented by the matrix

$$
\mathcal{B}=\frac{1}{\sqrt{2}}\left(\begin{array}{ll}
1 & i \\
i & 1
\end{array}\right),
$$

and the action of a phase plate can be written as

$$
\mathcal{P}(\theta)=\left(\begin{array}{cc}
1 & 0 \\
0 & e^{i \theta}
\end{array}\right),
$$

for any arbitrary real parameter $\theta$. One can easily see that any $\mathrm{U}(2)$ operator $W$ (up to an overall phase) can be realized using three phase plates and two BBSs as

$$
W=\mathcal{P}\left(\theta_{1}\right) \mathcal{B P}\left(\theta_{2}\right) \mathcal{B P}\left(\theta_{3}\right),
$$

which represents a Mach-Zehnder interferometer.
The polarization DoF forms a two-dimensional vector space spanned by two orthogonal polarization states. All the normal preserving operation on the polarization states span the SU(2) group. Some examples of such operations are halfwave plates (HWPs) and quarter-wave plates (QWPs), whose matrix form in the horizontal and vertical polarization states basis reads [41]

$$
\begin{gathered}
H(\theta)=e^{-i \pi / 2}\left(\begin{array}{cc}
\cos (2 \theta) & \sin (2 \theta) \\
\sin (2 \theta) & -\cos (2 \theta)
\end{array}\right) \\
Q(\theta)=e^{-i \pi / 4}\left(\begin{array}{cc}
\cos ^{2} \theta+i \sin ^{2} \theta & (1-i) \sin \theta \cos \theta \\
(1-i) \sin \theta \cos \theta & i \cos ^{2} \theta+\sin ^{2} \theta
\end{array}\right) .
\end{gathered}
$$

Here, $\theta$ is the angle between the fast axis of the WP and the horizontal axis. An arbitrary SU(2) operation on the polarization states can be realized using just one HWP and two QWPs [42-44].

Another interesting DoF of light is the OAM. Laguerre Gauss modes are the eigenmodes of the paraxial wave equation, and are represented by two indices $-\infty<\ell<\infty$ and $0 \leqslant p<\infty$. For $p=0$, $\ell$ characterizes the orbital angular momentum in the light beam (or single photon) [45]. Such states are known as OAM states of light and have infinitely many orthogonal states. Such states are highly sought after in quantum information and communication tasks due to their large Hilbert space. However, while in theory it is possible to implement any arbitrary transformation on OAMs, the methods to realize such transformations are not known.

\section{B. Quantum measurements}

The most general measurements in quantum theory are represented by a mathematical model in which the system of interest is coupled to a probe via some channel. Later on, a projective measurement is performed on the probe only. Due to the coupling between the probe and system, it is possible to infer information about the state of the latter by a projective measurement on the former. Such models are called quantum instruments [5-8].

In this paper, we look at a subclass of measurement models having a discrete number of outcomes in which the coupling channel between the probe and system is taken to be a unitary transformation. Such measurements are known as POVMs with immense scope of application in quantum information, as has been discussed earlier.

POVMs consist of a set of $N$ positive operators $\mathcal{M}=$ $\left\{E_{0}, E_{1}, \ldots, E_{N-1}\right\}$ known as effects acting on the $m$ dimensional Hilbert space $\mathcal{H}$, such that $E_{i} \geqslant 0 \forall i$ and $\sum_{i=0}^{N-1} E_{i}=\mathbb{1}$. There are three levels of descriptions of a quantum measurement which are the following:

(i) In the first level of description, only the statistics of the outcomes of the POVM are of interest with only the measurement effects $\left\{E_{i}\right\}$ being fully specified. The probability of the $i$ th measurement outcome is given by $p_{i} \equiv\left\langle E_{i}\right\rangle=\operatorname{Tr}\left(\rho E_{i}\right)$, i.e., the expectation value of the effect $E_{i}$.

(ii) The second level of description for quantum measurements involves the state update rules after the measurements. In this level, we specify the measurement operators $K_{i j}$ such that the $i$ th measurement outcome results in the transformation 
of the system's state

$$
\rho^{\prime}=\frac{\sum_{j} K_{i j} \rho K_{i j}^{\dagger}}{\operatorname{Tr}\left(K_{i j} \rho K_{i j}^{\dagger}\right)},
$$

and the probability for such event is given by $\operatorname{Tr}\left(K_{i j} \rho K_{i j}^{\dagger}\right)$. The relation between the effects $E_{i}$ and the measurement operators $K_{i j}$ reads

$$
E_{i}=\sum_{j} K_{i j}^{\dagger} K_{i j}
$$

(iii) In the third level of description, the detailed interaction between the system and the probe is specified along with the initial state of the probe. If the net effect of this interaction is represented by joint a unitary operator $U_{S P}$ acting on the system and probe, then the general transformation of the system plus probe is written as

$$
\rho_{S P}^{\prime}=U_{S P}\left(\rho_{S} \otimes \sigma_{P}\right) U_{S P}^{\dagger}
$$

Here the subscripts $S$ and $P$ stand for system and probe, respectively. $\sigma_{P}=\sum_{j} q_{j}\left|\phi_{j}\right\rangle\left\langle\phi_{j}\right|$ is the initial state of the probe where $\left|\phi_{j}\right\rangle$ and $q_{j}$ are the eigenvectors and eigenvalues of $\sigma_{P}$, and $\rho_{S}$ is the initial state of the system. The application of the unitary operator is followed by a projective measurement on the probe in an orthonormal basis $\{|i\rangle\}$. The measurement operators $K_{i j}$ and the unitary operator $U_{S P}$ is related as

$$
K_{i j}=\sqrt{q_{j}}\left\langle i\left|U_{S P}\right| \phi_{j}\right\rangle
$$

Hence, the measurement operators $K_{i j}$ contain information about the interaction between the system and the probe as well as the initial state of the probe.

For the special cases, when the probe is in a pure state initially, i.e., $\sigma_{B}=|0\rangle\langle 0|$ the measurement operators become $K_{i}=\left\langle i\left|U_{S P}\right| 0\right\rangle$ and the effects $\operatorname{read} E_{i}=K_{i}^{\dagger} K_{i}$. Such measurements are purity preserving measurements as in each measurement outcome the system state is a pure state if initially the system was in pure state.

Although the state update rule given in Eq. (6) is more general, for the rest of the paper we deal with measurement operators and their unitary equivalents for the choice of pure probe states. This makes some of the calculations easier, while keeping the scheme we present is general enough to also allow for mixed ancillary states.

Any POVM measurement described according to the second level with only the measurement operators $\left\{K_{i}\right\}$ specified, can be realized as a joint unitary evolution of the system state $\rho_{S}$ coupled with an ancilla initialized in the state $|0\rangle\left\langle\left. 0\right|_{A}\right.$, followed by a projective measurement on the latter with elements $\left\{\Pi_{i}=|i\rangle\langle i|\right\}$, where $\{|i\rangle\}$ is an orthonormal basis for the finite dimensional Hilbert space of the ancilla. If the joint unitary operator is $U$, then the probability to obtain the $i$ th outcome on the state $\rho_{A S}=|0\rangle\left\langle\left. 0\right|_{A} \otimes \rho_{S}\right.$ is then given by

$$
\begin{aligned}
P(i) & =\operatorname{Tr}\left(\Pi_{i} \otimes \mathbb{1}\left[U\left(|0\rangle\left\langle\left. 0\right|_{A} \otimes \rho_{S}\right) U^{\dagger}\right]\right)\right. \\
& =\operatorname{Tr}\left(K_{i}^{\dagger} K_{i} \rho_{S}\right),
\end{aligned}
$$

where $K_{i}=\langle i|U| 0\rangle$. Any unitary matrix $U$ of the form

$$
U=\left(\begin{array}{cccc}
K_{0} & A_{1,1} & \ldots & A_{1, N-1} \\
K_{1} & A_{1,1} & \ldots & A_{2, N-1} \\
\vdots & \vdots & \ddots & \vdots \\
K_{N-1} & A_{N, 1} & \ldots & A_{N \ldots N-1}
\end{array}\right)
$$

will result in the measurement operators $K_{i}$. Since we are working in the second level of description of POVMs, the matrix $U$ is not unique and the $m \times m$ matrices $A_{i, j}$ can be chosen arbitrarily as long as $U$ is unitary.

Projective measurements are a special case of POVMs where $E_{i}$ 's are one-dimensional projectors with an additional constraint $\operatorname{Tr}\left(E_{i} E_{j}\right)=\delta_{i j}$, which states that the outcomes form an orthonormal basis. Unlike a nondegenerate projective measurement, the number of outcomes in a POVM need not be equal to the dimension of Hilbert space of the system and can also be continuous. For example, a measurement of the direction of spin-1/2 particles is a POVM with a continuous spectrum of outcomes. Moreoever, such POVMs can be transformed into a random choice of measurements with a finite number of outcomes [46]. However, dealing with measurements having a continuous set of outcomes including homodyne and heterodyne measurements is beyond the scope of this paper, while we primarily focus on discrete outcome measurements only. We implement these POVMs using unitary transformations and projective measurements.

An important class of POVMs is called SIC-POVM. The measurement effects for SIC-POVMs are proportional to onedimensional projectors. For a single qubit the SIC-POVM consists of the operators $\mathcal{M}=\left\{\frac{1}{2} \Pi_{0}, \frac{1}{2} \Pi_{1}, \frac{1}{2} \Pi_{2}, \frac{1}{2} \Pi_{3}\right\}$, where $\Pi_{i}=\left|\psi_{i}\right\rangle\left\langle\psi_{i}\right|$, such that

$$
\operatorname{Tr}\left(\Pi_{i} \Pi_{j}\right)=\frac{2 \delta_{i j}+1}{3}, \quad \forall i, j \in\{0,1,2,3\} .
$$

The corresponding measurement operators for this SICPOVM can be chosen as $K_{i}=\frac{1}{\sqrt{2}} \Pi_{i}$. SIC-POVMs are known to be important for many quantum information processing tasks $[47,48]$ and quantum measurements [19,49]. For example, one can perform a full state tomography for a single-qubit state $\rho$ by estimating the probabilities $p_{i}=\operatorname{Tr}\left(\rho K_{i}^{\dagger} K_{i}\right)$ of various outcomes of the SIC-POVM given above. From these outcome probabilities and information about the SIC-POVM effects $\mathcal{M}$ one can reconstruct the density operator $\rho$.

\section{CS decomposition}

CS decomposition is a powerful method to decompose an arbitrary unitary operator $U$ into smaller unitaries and cosinesine $(C)$ matrices [15]. The most notable application of this decomposition is in the optical systems where the smaller matrices correspond to the operations on the internal degree of freedom of light such as polarization and OAM and the CS matrices correspond to generalized Mach-Zehnder interferometers.

CS decomposition states that an arbitrary $(m+n) \times$ $(m+n)$ unitary matrix $U_{m+n}(n \geqslant m)$ can be decomposed into $n \times n$ and $m \times m$ unitaries and a CS matrix as [15]

$$
U_{m+n}=\left(\begin{array}{cc}
L_{m} & 0 \\
0 & L_{n}^{\prime}
\end{array}\right)\left(\mathcal{S}_{2 m} \oplus \mathbb{1}_{n-m}\right)\left(\begin{array}{cc}
R_{m}^{\dagger} & 0 \\
0 & R_{n}^{\dagger \dagger}
\end{array}\right),
$$




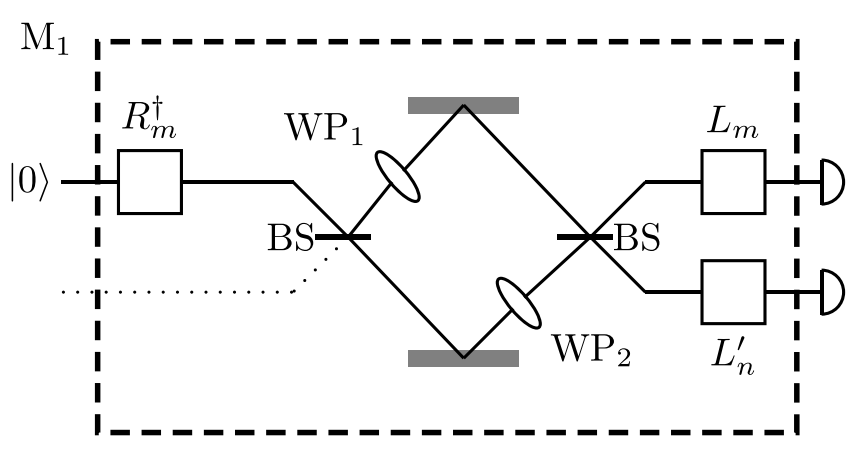

FIG. 1. An optical setup to implement a two-outcome POVM. Here the two wires represent the two spatial modes and the internal degrees of freedom are inherent in each wire. The operations $R_{m}^{\dagger}, L_{m}$, and $L_{m}^{\prime}$ act only on the internal modes in their respective spatial modes. The $\mathcal{S}_{2 m}$ operator is realized using two BBSs and two WPs. Finally, one performs the intensity measurement on the two spatial modes which yields the probability of the two measurement outcomes. This optical setup can implement a discrete valued twooutcome POVM only.

where $\mathcal{S}_{2 m}$ is the CS matrix given as

$$
\mathcal{S}_{2 m}=\left(\begin{array}{cc}
C_{m} & -S_{m} \\
S_{m} & C_{m}
\end{array}\right) .
$$

Here $C_{m}=\operatorname{diag}\left\{\cos \theta_{1}, \cos \theta_{2}, \ldots, \cos \theta_{m}\right\} \quad$ and $S_{m}=$ $\operatorname{diag}\left\{\sin \theta_{1}, \sin \theta_{2}, \ldots, \sin \theta_{m}\right\}$. The matrix $\mathcal{S}_{2 m}$ can be further simplified as

$$
\mathcal{S}_{2 m}=\left(B_{2} \otimes \mathbb{1}_{m}\right)\left(\Theta_{m} \oplus \Theta_{m}^{\dagger}\right)\left(B_{2}^{\dagger} \otimes \mathbb{1}_{m}\right),
$$

where

$$
\begin{gathered}
B_{2}=\frac{1}{\sqrt{2}}\left(\begin{array}{ll}
1 & i \\
i & 1
\end{array}\right), \\
\Theta_{m}=\operatorname{diag}\left(e^{i \theta_{1}}, e^{i \theta_{2}}, \ldots, e^{i \theta_{m}}\right) .
\end{gathered}
$$

Since $L_{m}, L_{n}^{\prime}, R_{m}^{\dagger}, R_{n}^{\prime \dagger}$ are unitary, the CS decomposition can be applied iteratively to break the unitaries into smaller dimensional unitaries, as is explained in detail in Ref. [15]. In optical systems, $B_{2} \equiv \mathcal{B}$ represents the BBS and the $\Theta_{m}$ matrix corresponds to the phase operation on the internal degree of freedom. For $m=2$, it can be seen that the phase operation corresponds to a WP. Hence, the CS matrix represents a generalized Mach-Zehnder interferometer. The unitary operations $L_{m}, L_{n}^{\prime}, R_{m}^{\dagger}, R_{n}^{\prime \dagger}$ are the operators acting on the internal degrees of freedom of light. These unitaries are applied in a particular path of beam. Hence, they take on the form of control unitaries and are of the form

$$
L=|0\rangle\left\langle 0\left|\otimes L_{m}+\right| 1\right\rangle\langle 1| \otimes L_{n}^{\prime},
$$

with a similar control operator for the unitaries $R_{m}^{\dagger}$ and $R_{n}^{\prime \dagger}$. As can be seen the spatial mode controls which unitary operation acts on the internal degrees of freedom. Such control operations can be easily handled on optical systems, where the corresponding operators can simply act on the photons in different modes locally as shown in Fig. 1.

\section{RESULTS}

In this section, we describe the scheme to implement an arbitrary POVM on optical systems based on the second level of description of quantum measurements in which the measurement operators are specified. Here the internal DoFs of photons span the Hilbert space for the systems and the spatial modes serve as the ancilla. We use Naimark dilation theorem and CS decomposition to implement the POVM. In order to implement an $n$-outcome POVM we require $n-1$ BS setups along with unitary transformations on the internal DoFs in each of the spatial modes, irrespective of the dimension of the system. As an example, we present a scheme single-shot state tomography of a quantum state.

We start with the simplest case of POVM, i.e., a twooutcome POVM in Sec. III A and generalize this result to $n$-outcome POVM in Sec. III B.

\section{A. Two-outcome POVMs}

Consider a two-outcome POVM specified according to the second level of description of measurement with measurement operators $\left\{K_{0}, K_{1}\right\}$ acting on an $m$-dimensional Hilbert space. Following the discussion in Sec. II B we can write the unitary acting on the system and a two-dimensional ancilla as

$$
U=\left(\begin{array}{ll}
K_{0} & A \\
K_{1} & B
\end{array}\right)
$$

where $A$ and $B$ are appropriately chosen complex matrices of dimension $m \times m$ such that the matrix $U$ is unitary. The matrix $U$ is acting on the Hilbert space $\mathcal{H}_{a} \otimes \mathcal{H}_{s}$, where $a$ and $s$ stand for ancilla and system, respectively.

Our aim is to design an optical setup to implement the unitary operator $U$ and the desired projective measurements. If we consider the spatial modes of a photon as the ancilla and the internal modes such as polarization, orbital angular momentum, or frequency modes as the system states, then using CS decomposition we can decompose $U$ into BS operations on the spatial modes and unitary operations on the internal modes.

The CS decomposition of the operator $U$ reads

$$
U=\left(\begin{array}{cc}
L_{m} & 0 \\
0 & L_{m}^{\prime}
\end{array}\right)\left(\begin{array}{cc}
C_{m} & -S_{m} \\
S_{m} & C_{m}
\end{array}\right)\left(\begin{array}{cc}
R_{m}^{\dagger} & 0 \\
0 & R_{m}^{\prime \dagger}
\end{array}\right) .
$$

Here $L_{m}, L_{m}^{\prime}, R_{m}^{\dagger}$ are $m \times m$ unitary matrices acting on the internal modes of the photon and $C_{m}, S_{m}$ are cosine and sine matrices [see Eq. (14)]. From here we can write

$$
\begin{aligned}
& K_{0}=L_{m} C_{m} R_{m}^{\dagger}, \\
& K_{1}=L_{m}^{\prime} S_{m} R_{m}^{\dagger} .
\end{aligned}
$$

Equation (21) is simply a singular value decomposition of the measurement operator $K_{i}$, which can be solved efficiently to get $L_{m}, L_{m}^{\prime}, R_{m}^{\dagger}$ operators and the $C_{m}, S_{m}$ matrices. Furthermore, the choice of $A$ and $B$ matrices reflects in choosing the unitary $R_{m}^{\prime \dagger}$. While working in the paradigm of second level of description of quantum measurements in which the unitary operation is not completely specified, this freedom can be exploited to simplify the setup by having $R_{m}^{\prime \dagger}=\mathbb{1}$. We further discuss the case of first and third level of description of quantum measurements in Sec. III B. 
The matrix $\mathcal{S}_{2 m}$, constructed from $C_{m}$ and $S_{m}$ can be further decomposed into BS and phase shift transformations as given in Eq. (15).

The local unitary matrices $L_{m}, L_{m}^{\prime}, R_{m}^{\dagger}$ can be implemented on optical systems and for the case of polarization degrees of freedom, it requires just one half WP and two quarter WPs mounted coaxially $[42,43]$.

Finally, a projective measurement is implemented on the ancillary modes. This can be viewed as detection of a photon in one or the other spatial mode corresponding to the two outcomes of the POVM. The resultant frequency of clicks of each detector can then be used to simulate the statistics of the POVM. This way it is possible to implement a measurement according to the first level of description. However, it is not always necessary to implement a photon detection on both of the spatial modes. The updated state after the application of the POVM can be extracted corresponding to a particular outcome by postselection. As an example, the updated state corresponding to a measurement operator $K_{i}$, given by Eq. (6), can be obtained by not placing any detectors on this mode, while having photon detections on the modes $j \neq i$. Whenever the detectors on the modes $j \neq i$ do not click, we are assured to get the required updated state in the $i$ th mode. Using this approach it is thus possible to implement a measurement according to the second and third level of description.

Hence, an arbitrary two-outcome POVM can be implemented in optical systems. A schematic diagram to implement these POVM is given in Fig. 1.

It is to be noted that spatial degrees of freedom (as denoted by wires in Fig. 1) are taken as ancilla while internal degrees of freedom correspond to the system as in Eq. (10). The initialization of the ancilla state to $|0\rangle\langle 0|$ implies that the system is injected into the setup through the upper spatial mode. For a special choice of mixed states of ancilla $\sigma_{B}=\sum_{j=0}^{1} p_{j}|j\rangle\langle j|$, where the eigenstates $|j\rangle, j \in\{0,1\}$ correspond to the upper and lower spatial modes, the system is injected through the upper spatial mode with probability $p_{0}$ and the lower spatial mode with probability $p_{1}=1-p_{0}$. It should be noted that a mixed state can also be prepared probabilistically using our technique described in Sec. IV B. This way we can implement a general quantum instrument.

\section{B. $n$-outcome POVMs}

In this section, we generalize the results on the previous section to implement an $n$-outcome POVM on optical systems. Consider a POVM with $n$ number of $m$-dimensional measurement operators $\left\{K_{0}, K_{1}, \ldots, K_{n-1}\right\}$. The corresponding $\mathrm{nm} \times \mathrm{nm}$ unitary operator $U$ is given in Eq. (11) with $m \times m$ complex matrices $A_{i, j}$ chosen such that $U$ is unitary.

Similar to the two-outcome case, we can decompose the matrix $U$ as

$$
U=\left(\begin{array}{cc}
L_{m}^{(1)} & 0 \\
0 & L_{m(n-1)}^{\prime(1)}
\end{array}\right)\left(\mathcal{S}_{2 m}^{(1)} \oplus \mathbb{1}_{m(n-2)}\right)\left(\begin{array}{cc}
R_{m}^{(1) \dagger} & 0 \\
0 & R_{m(n-1)}^{(1) \dagger}
\end{array}\right),
$$

where the unitary operators $L_{m}^{(1)}$ and $R_{m}^{(1) \dagger}$ are of dimension $m$, while $L_{m(n-1)}^{\prime(1)}$ and $R_{m(n-1)}^{\prime(1) \dagger}$ are of $m(n-1)$ dimension, and $\mathcal{S}_{2 m}^{(1)}$ is a $2 \times 2$ block matrix where each block is an $m \times m$ diagonal matrix consisting of cosine and sine as given in Eq. (14). The superscript (1) denotes the first iteration of CS decomposition.

Since $A_{i, j}$ are chosen arbitrarily, this gives us freedom to choose any $m(n-1)$-dimensional unitary for $R_{m(n-1)}^{(1)}$. For the sake of simplicity, we choose $R_{m(n-1)}^{(1)}=\mathbb{1}$. With this choice of $R_{m(n-1)}^{(1)}$, we get

$$
\begin{gathered}
K_{0}=L_{m}^{(1)} C_{m}^{(1)} R_{m}^{(1) \dagger}, \\
\left(\begin{array}{c}
K_{1} \\
K_{2} \\
\vdots \\
K_{n-1}
\end{array}\right)=L_{m(n-1)}^{(1)}\left(\begin{array}{c}
S_{m}^{(1)} \\
\mathcal{O} \\
\vdots \\
\mathcal{O}
\end{array}\right) R_{m}^{(1) \dagger} .
\end{gathered}
$$

Here $\mathcal{O}$ is an $m \times m$ null matrix. From there it is clear that only the first $m$ number of columns of $L_{m(n-1)}^{\prime(1)}$ will contribute in $K_{i}$ measurement operators; therefore, the rest of the columns can be chosen arbitrarily. In this sense the problem is similar to the one with $U$ matrix, but now we have $n-1$ measurement operators. We can further use CS decomposition for the $L_{m(n-1)}^{\prime(1)}$ operator to find $m \times m$ unitary operators $L_{m}^{(2)}$ and $R_{m}^{(2) \dagger}$ such that $K_{1}=L_{m}^{(2)} C_{m}^{(2)} R_{m}^{(2) \dagger} S_{m}^{(1)} R_{m}^{(1) \dagger}$, an $m(n-2)$-dimensional unitary operator $R_{m(n-2)}^{\prime(2) \dagger}=\mathbb{1}$, and an $m(n-2)$-dimensional $L_{m(n-2)}^{\prime(2)}$. Recursively CS decompos$\operatorname{ing} L_{m(n-j)}^{\prime(j)}$ unitary operators in the $j$ th iteration, we can get

$$
\begin{aligned}
K_{0} & =L_{m}^{(1)} C_{m}^{(1)} R_{m}^{(1) \dagger} \\
K_{1} & =L_{m}^{(2)} C_{m}^{(2)} R_{m}^{(2) \dagger} S_{m}^{(1)} R_{m}^{(1) \dagger} \\
K_{2} & =L_{m}^{(3)} C_{m}^{(3)} R_{m}^{(3) \dagger} C_{m}^{(2)} R_{m}^{(2) \dagger} S_{m}^{(1)} R_{m}^{(1) \dagger} \\
\vdots & \\
K_{n-2} & =L_{m}^{(n-1)} C_{m}^{(n-1)} R_{m}^{(n-1) \dagger} \ldots S_{m}^{(1)} R_{m}^{(1) \dagger}, \\
K_{n-1} & =L_{m}^{(n)} S_{m}^{(n-1)} R_{m}^{(n-1) \dagger} \ldots S_{m}^{(1)} R_{m}^{(1) \dagger} .
\end{aligned}
$$

Since each of the $\mathcal{S}^{(j)}$ represents a generalized MachZehnder interferometer which can be realized using two balanced BS and two diagonal unitaries on the internal states of photons, an $n$-outcome POVM can be decomposed into $2 n$ general unitary operations and $2 n-2$ diagonal unitaries on the internal states of the photons and $2 n-2$ BBS.

In order to solve Eq. (25), we can convert these equations into singular value decompositions as follows: the first equation is already a singular value decomposition of $K_{0}$ which yields $L_{m}^{(1)}, R_{m}^{(1) \dagger}$ and the diagonal matrices $C_{m}^{(1)}$ and $S_{m}^{(1)}=$ $\sqrt{1-\left(C_{m}^{(1)}\right)^{2}}$. Using this we can rewrite the second equation as

$$
K_{1}\left(S_{m}^{(1)} R_{m}^{(1) \dagger}\right)^{-1}=L_{m}^{(2)} C_{m}^{(2)} R_{m}^{(2) \dagger},
$$

which is a singular value decomposition which yields $L_{m}^{(2)}, R_{m}^{(2) \dagger}$, and $C_{m}^{(2)}$. Similarly, one can find all the other matrices and construct the optical setup. It can be seen that for decomposing any $K_{i}$, it is required to evaluate a simple singular value decomposition problem.

A schematic diagram to implement a four-outcome POVM for $m=2$ is given in Fig. 2 which can be extended in a similar fashion to a higher number of outcomes as desired. As can be seen the module to implement a two-outcome POVM appears 


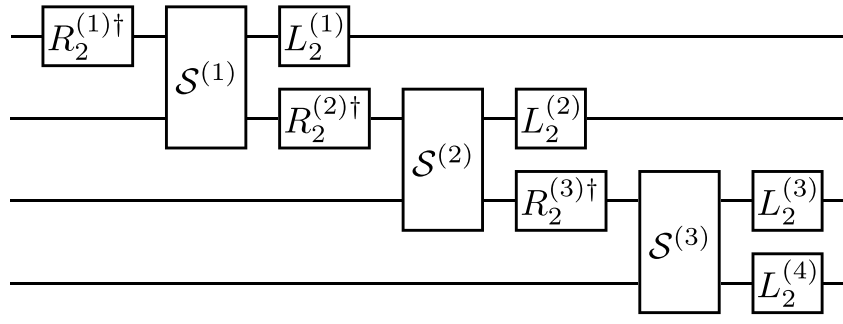

FIG. 2. A schematic diagram to implement a four-outcome POVM which can be easily generalized for $n$ outcomes. Here the wires represent spatial modes and the internal DoFs are inherent in each wire. The module to implement a two-outcome POVM as shown in Fig. 1 is seen to appear between two different modes more than once in this schematic. This setup can implement a discrete valued four-outcome POVM only.

more than once in the schematic. A numerical code for the same can be found at [50].

The most crucial step in solving Eq. (25) is taking the inverse of matrix $\left(S_{m}^{(1)} R_{m}^{(1) \dagger}\right)$. For the case of $n=2$, this problem does not arise. However, for $n>2$, the situation is a little nontrivial. For the case of two-level systems, i.e., $m=2$, the operator $S_{2}^{(1)}$ is noninvertible only if $E_{0}=K_{0}^{\dagger} K_{0}$ is a rankone projection. For $m=2$ and $n>2$, not all the $E_{i}$ 's can be rank-one projectors, as can be seen from the condition $\sum_{i} E_{i}=\sum_{i} K_{i}^{\dagger} K_{i}=\mathbb{1}$. Therefore, we can always find at least $n-2$ number of measurement operators for which the $S_{2}$ is invertible. Therefore, for these systems Eq. (25) can be solved exactly.

For $m>2$, solving Eq. (25) may not always be possible. For example, for $m=3$ the projective measurement contains three measurement operators each of which are one-dimensional projectors. Therefore, the first equation in Eq. (25) will yield a rank-one $C_{m}^{(1)}$ matrix and rank-two $S_{m}^{(1)}$ matrix, which is noninvertible. Therefore, even for projective measurements it is difficult to solve this equation using the method prescribed above.

A numerical method can be used for the $m>2$ case. In this method, we construct the unitary operator $U$ using the given measurement operators $\left\{K_{i}\right\}$ and choose matrices $A_{i j}$ randomly such that the matrix $U$ is unitary. This can easily be ensured by using Gram-Schmidt orthogonalization. Using CS decomposition numerically on the operator $U$ we can obtain all the optical components required to experimentally realize it. Schematically, the decomposition looks as shown in Fig. 3. From Eq. (25) it is known that only the rightmost set of $L_{m}^{(i)}$ operators, $\mathcal{S}_{m}^{(i)}$ operators, and $R_{m}^{(i) \dagger}$ operators contribute to the measurement operators $K_{i}$. All the rest of the operators in the decomposition can be chosen arbitrarily, without affecting $K_{i}$. Therefore, for convenience we can choose the rest of the operators to be identity.

The protocol so presented can accommodate the three levels of description of a quantum measurement as described in Sec. II B. The aforementioned analysis for two- and noutcome POVMs is presented keeping in mind the second level of description, in which the measurement operators are specified. The schemes presented in Figs. 1 and 2 suffice for this case.

For a description of the first level, we are given only the effects $\left\{E_{i}=K_{i}^{\dagger} K_{i}\right\}$; there can be infinitely many measurement operators resulting in the same $E_{i}$. Any measurement operator $\tilde{K}_{i}=W K_{i}$ where $W^{\dagger} W=\mathbb{1}$ will also yield $E_{i}=\tilde{K}_{i}^{\dagger} \tilde{K}_{i}=$ $K_{i}^{\dagger} K_{i}$. In this case we are free to choose the simplest possible $\tilde{K}_{i}$ for our purpose. Using this freedom we can remove the $L_{m}^{(i)}$ unitary operators from the setup. Therefore, we need only $n$ number of general unitaries, $2 n-2$ diagonal unitaries, and $2 n-2$ BBSs. This greatly simplifies our experimental setup.

For the third level of description, the unitary operator is completely specified and therefore, we no longer have the freedom to choose $R_{m}^{\prime \dagger}=\mathbb{1}$. This complicates the setup as more unitaries have to be implemented now. A schematic diagram to implement a four-outcome POVM according to the third level of description is given in Fig. 2, where the grayed out components can no longer be set to identity. As is evident from the discussion, the complexity of the experimental setup increases with the level of description of the POVM.

\section{APPLICATIONS}

In this section we detail some applications of CS decomposition to implement POVMs on optical systems and perform their cost analysis in terms of the required number of optical elements.

\section{A. Single-shot quantum state tomography of photonic qubit}

In general, complete state tomography of a two-level system requires estimating the expectation value of three

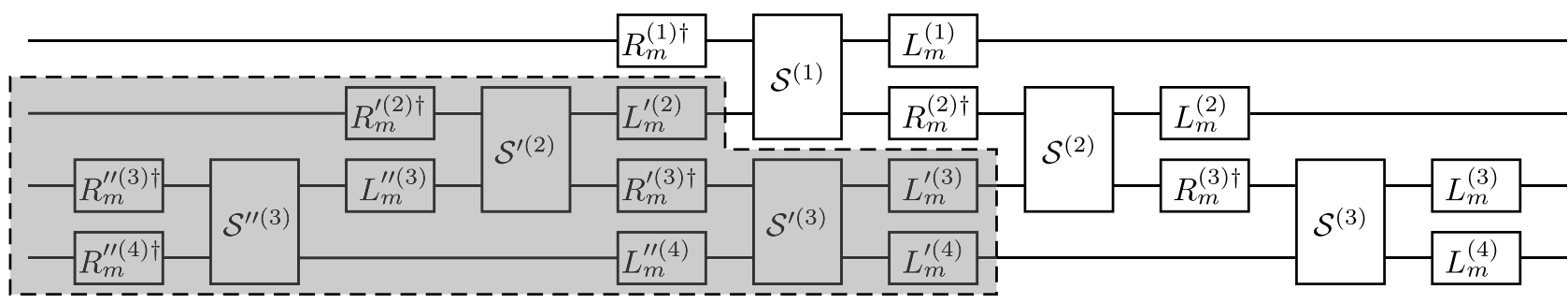

FIG. 3. A schematic diagram to experimentally realize an arbitrary four-outcome POVM using the Gram-Schmidt orthogonalization method for the nontrivial cases in the $m>2$ scenario. The wires represent spatial modes of light, while the internal DoFs are inherent in each wire. All the optical elements appearing in the grayed out box can be set to identity without compromising either the measurement operators or the CS decomposition when either the first or second level of description of a quantum measurement is specified. The resultant decomposition then resembles the one given in Fig. 2. However, that is no longer the case when dealing with the third level, when the elements in the grayed box can be set to identity. This optical setup can implement discrete valued POVMs only. 
noncommuting observables. This can be done using three measurement settings. However, changing the experimental setting can cause errors and misalignment, which can result in inaccurate outcomes. Single-shot quantum state tomography is a technique where a single experimental setup can be used to estimate the state of a quantum system. This technique often requires measurements involving POVMs. One can use SICPOVM to perform single-shot state tomography. However, generally it is difficult to perform four-outcome POVMs on optical systems.

In order to implement a SIC-POVM on polarization qubits using our scheme we need six BSs, six WPs, and seven unitaries acting on spatial modes. The schematics of this setup is given in Fig. 2. The $2 \times 2$ unitary operators $L_{2}^{(i)}$ and $R_{2}^{(i)}$ can be calculated for a given SIC-POVM using Eq. (25) given in Sec. III B. The low number of BSs and WPs required in our scheme makes it one of most viable options to implement single-shot quantum state tomography in a cost-effective manner.

\section{B. Preparing arbitrary mixed states of a quantum system}

Another interesting application of optical implementation of arbitrary POVM is the preparation of an arbitrary mixed state in optical systems. Mixed states are important to calibrate the experimental setups. Furthermore, the mixed states have a fundamental uncertainty as they do not retain the information about the preparation basis. Here we show how POVMs can be used to prepare an arbitrary given state $\rho$ for $m$-dimensional quantum systems.

For this purpose we need a setup for a two-outcome POVM and a maximally mixed state $\rho_{r}=\mathbb{1} / m$ as the input in the optical setup as shown in Fig. 1. The measurement operators we choose are $K_{0}=\sqrt{\rho / \lambda_{0}}$ and $K_{1}=\sqrt{1-\rho / \lambda_{0}}$ where $\lambda_{0}$ is the largest eigenvalue of the state $\rho$. One can check that $K_{0}^{\dagger} K_{0}+K_{1}^{\dagger} K_{1}=\mathbb{1}$.

Using the technique described in Sec. III A we can decompose the POVM into simple unitaries acting on internal and external DoFs. The choice of local unitaries and phase shifters will depend on the state $\rho$ that is being prepared and can be easily calculated using our prescription. At this stage no final projective measurement as described in Sec. III A has been made on any of the output modes. Consequently, the output states in each of the two modes can be seen to correspond to $K_{0} \rho_{r} K_{0}^{\dagger}=\rho /\left(m \lambda_{0}\right)$ with probability $p_{0}=1 /\left(m \lambda_{0}\right)$ in the upper spatial mode and $E_{1}=K_{1} \rho_{r} K_{1}^{\dagger}=\left(\mathbb{1}-\rho / \lambda_{0}\right) / m$ with probability $p_{1}=1-1 /\left(m \lambda_{0}\right)$ in the lower spatial mode. Hence, the state of the internal degree of freedom in the upper spatial mode is the desired state $\rho$ before performing the measurement.

Interestingly, for the case of $m=2$ the eigenvalues of the density operator $\rho$ are of the form $\lambda, 1-\lambda$, for $0 \leqslant \lambda \leqslant 1$. The spectrum of $\mathbb{1}-\rho$ is the same. Therefore, we can find a unitary transformation $V$ such that $V(\mathbb{1}-\rho) V^{\dagger}=\rho$. Hence, using an appropriate transformation $V$ on the lower spatial mode, we can transform the outcome into $\rho$ too. This gives us a $100 \%$ success rate for creating an arbitrary single-qubit mixed state.

\section{CONCLUSION}

In this paper we detailed a protocol to implement any arbitrary POVMs on internal degrees of freedom of a light beam. The basis of our protocol lies in CS decomposition which can be used to decompose any complicated unitary matrix into simpler ones. Applying Naimark's dilation theorem in conjunction with CS decomposition it is possible to find a unitary matrix corresponding to any POVM which can then be further decomposed. Using our method any $n$ outcome POVM acting on an arbitrary dimension Hilbert space can be experimentally implemented with a $100 \%$ success rate with a far lower number of optical elements than the current techniques.

Furthermore, our technique can quite easily accommodate the three levels of description of quantum measurements by modifying the optical setup by incorporating more (or less) local unitaries acting on the internal DoF. We show that the complexity of the experimental setup increases with the level of description.

Since it is quite hard to experimentally implement POVMs with arbitrary number of outcomes, our scheme makes it possible to study their effects and applications in scenarios like local filtering, state tomography [19,20], quantum key distribution [23], and quantum nonclassicality [27,51], where they are known to play an important role.

Further, the techniques discussed in this paper are experimentally feasible with the current technology and can be readily implemented.

\section{ACKNOWLEDGMENTS}

J.S. acknowledges support from CSIR-UGC NET, India. J.S. and A. acknowledge the funding from the project DST/ICPS/QuST/Theme-1/2019/General Project No. Q-68. S.K.G. acknowledges the financial support from Interdisciplinary Cyber Physical Systems (ICPS) programme of the Department of Science and Technology, India (Grant No. DST/ICPS/QuST/Theme-1/2019/12).
[1] M. Nielsen and I. Chuang, Quantum Computation and Quantum Information (Cambridge University Press, Cambridge, UK, 2010).

[2] J. A. Bergou, Quantum state discrimination and selected applications, J. Phys.: Conf. Ser. 84, 012001 (2007).

[3] C. Gerry, P. Knight, and P. Knight, Introductory Quantum Optics (Cambridge University Press, Cambridge, UK, 2005).

[4] M. Scully and M. Zubairy, Quantum Optics (Cambridge University Press, Cambridge, UK, 1997).
[5] A. S. Holevo, Statistical Structure of Quantum Theory (Springer, New York, 2001).

[6] H. Wiseman and G. Milburn, Quantum Measurement and Control (Cambridge University Press, Cambridge, UK, 2010).

[7] T. Heinosaari and M. Ziman, The Mathematical Language of Quantum Theory: From Uncertainty to Entanglement (Cambridge University Press, Cambridge, UK, 2011).

[8] P. Busch, P. Lahti, J. P. Pellonpää, and K. Ylinen, Quantum Measurement (Springer International Publishing, Cham, 2016). 
[9] A. Peres, Quantum Theory: Concepts and Methods, Fundamental Theories of Physics (Springer, New York, 1995).

[10] H. E. Brandt, Positive operator valued measure in quantum information processing, Am. J. Phys. 67, 434 (1999).

[11] F. Hirsch, M. T. Quintino, J. Bowles, and N. Brunner, Genuine Hidden Quantum Nonlocality, Phys. Rev. Lett. 111, 160402 (2013).

[12] T. Vértesi and E. Bene, Two-qubit Bell inequality for which positive operator-valued measurements are relevant, Phys. Rev. A 82, 062115 (2010).

[13] D. Petz and L. Ruppert, Optimal quantum-state tomography with known parameters, J. Phys. A: Math. Theor. 45, 085306 (2012).

[14] Y. Ota, S. Ashhab, and F. Nori, Implementing general measurements on linear optical and solid-state qubits, Phys. Rev. A 85, 043808 (2012).

[15] I. Dhand and S. K. Goyal, Realization of arbitrary discrete unitary transformations using spatial and internal modes of light, Phys. Rev. A 92, 043813 (2015).

[16] J. Bae and L.-C. Kwek, Quantum state discrimination and its applications, J. Phys. A: Math. Theor. 48, 083001 (2015).

[17] B. He and J. A. Bergou, A general approach to physical realization of unambiguous quantum-state discrimination, Phys. Lett. A 356, 306 (2006).

[18] M. Szczykulska, T. Baumgratz, and A. Datta, Multi-parameter quantum metrology, Adv. Phys.: X 1, 621 (2016).

[19] J. M. Renes, R. Blume-Kohout, A. J. Scott, and C. M. Caves, Symmetric informationally complete quantum measurements, J. Math. Phys. 45, 2171 (2004).

[20] A. J. Scott, Tight informationally complete quantum measurements, J. Phys. A: Math. Gen. 39, 13507 (2006).

[21] T. Konrad, A. Rouillard, M. Kastner, and H. Uys, Robust control of quantum systems by quantum systems, Phys. Rev. A 104, 052614 (2021).

[22] F. Verstraete, J. Dehaene, and B. DeMoor, Local filtering operations on two qubits, Phys. Rev. A 64, 010101(R) (2001).

[23] J. Singh, S. Ghosh, Arvind, and S. K. Goyal, Role of Bell$\mathrm{CHSH}$ violation and local filtering in quantum key distribution, Phys. Lett. A 392, 127158 (2021).

[24] M. Mishra, R. Sengupta, and Arvind, Increasing distillable key rate from bound entangled states by using local filtration, Phys. Rev. A 102, 032415 (2020).

[25] F. Hirsch, M. T. Quintino, and N. Brunner, Quantum measurement incompatibility does not imply Bell nonlocality, Phys. Rev. A 97, 012129 (2018).

[26] R. Uola, T. Moroder, and O. Gühne, Joint Measurability of Generalized Measurements Implies Classicality, Phys. Rev. Lett. 113, 160403 (2014).

[27] R. W. Spekkens, Contextuality for preparations, transformations, and unsharp measurements, Phys. Rev. A 71, 052108 (2005).

[28] M. D. Mazurek, M. F. Pusey, R. Kunjwal, K. J. Resch, and R. W. Spekkens, An experimental test of noncontextuality without unphysical idealizations, Nat. Commun. 7, ncomms11780 (2016).

[29] F. E. Becerra, J. Fan, and A. Migdall, Implementation of generalized quantum measurements for unambiguous discrimination of multiple non-orthogonal coherent states, Nat. Commun. 4, 2028 (2013).
[30] L. Bartůšková, A. Černoch, J. Soubusta, and M. Dušek, Programmable discriminator of coherent states: Experimental realization, Phys. Rev. A 77, 034306 (2008).

[31] Z. E. D. Medendorp, F. A. Torres-Ruiz, L. K. Shalm, G. N. M. Tabia, C. A. Fuchs, and A. M. Steinberg, Experimental characterization of qutrits using symmetric informationally complete positive operator-valued measurements, Phys. Rev. A 83, 051801(R) (2011).

[32] Z.-W. Wang, X.-F. Zhou, Y.-F. Huang, Y.-S. Zhang, X.-F. Ren, and G.-C. Guo, Experimental Entanglement Distillation of Two-Qubit Mixed States Under Local Operations, Phys. Rev. Lett. 96, 220505 (2006).

[33] S. E. Ahnert and M. C. Payne, General implementation of all possible positive-operator-value measurements of single-photon polarization states, Phys. Rev. A 71, 012330 (2005).

[34] S. E. Ahnert and M. C. Payne, All possible bipartite positiveoperator-value measurements of two-photon polarization states, Phys. Rev. A 73, 022333 (2006).

[35] B. He, J. A. Bergou, and Z. Wang, Implementation of quantum operations on single-photon qudits, Phys. Rev. A 76, 042326 (2007).

[36] Y.-Y. Zhao, N.-K. Yu, P. Kurzyński, G.-Y. Xiang, C.-F. Li, and G.-C. Guo, Experimental realization of generalized qubit measurements based on quantum walks, Phys. Rev. A 91, 042101 (2015).

[37] Y. S. Yordanov and C. H. W. Barnes, Implementation of a general single-qubit positive operator-valued measure on a circuit-based quantum computer, Phys. Rev. A 100, 062317 (2019).

[38] M. Oszmaniec, L. Guerini, P. Wittek, and A. Acín, Simulating Positive-Operator-Valued Measures with Projective Measurements, Phys. Rev. Lett. 119, 190501 (2017).

[39] M. Oszmaniec, F. B. Maciejewski, and Z. Puchała, Simulating all quantum measurements using only projective measurements and postselection, Phys. Rev. A 100, 012351 (2019).

[40] M. Reck, A. Zeilinger, H. J. Bernstein, and P. Bertani, Experimental Realization of Any Discrete Unitary Operator, Phys. Rev. Lett. 73, 58 (1994).

[41] R. C. Jones, A new calculus for the treatment of optical systems. I. Description and discussion of the calculus, J. Opt. Soc. Am. 31, 488 (1941).

[42] R. Simon and N. Mukunda, UniversalSU(2) gadget for polarization optics, Phys. Lett. A 138, 474 (1989).

[43] R. Simon and N. Mukunda, Minimal three-component SU(2) gadget for polarization optics, Phys. Lett. A 143, 165 (1990).

[44] Arvind, G. Kaur, and G. Narang, Optical implementations, oracle equivalence, and the Bernstein-Vazirani algorithm, J. Opt. Soc. Am. B 24, 221 (2007).

[45] L. Allen, M. W. Beijersbergen, R. J. C. Spreeuw, and J. P. Woerdman, Orbital angular momentum of light and the transformation of Laguerre-Gaussian laser modes, Phys. Rev. A 45, 8185 (1992).

[46] G. Chiribella, G. M. D’Ariano, and D. Schlingemann, How Continuous Quantum Measurements in Finite Dimensions Are Actually Discrete, Phys. Rev. Lett. 98, 190403 (2007).

[47] C. M. Caves, C. A. Fuchs, and R. Schack, Unknown quantum states: The quantum de Finetti representation, J. Math. Phys. 43, 4537 (2002). 
[48] C. A. Fuchs and M. Sasaki, Squeezing quantum information through a classical channel: Measuring the "quantumness" of a set of quantum states, Quantum Inf. Comput. 3, 377 (2003).

[49] M. Appleby, S. Flammia, G. McConnell, and J. Yard, Sics and algebraic number theory, Found. Phys. 47, 1042 (2017).
[50] J. Singh, Optical POVM, https://github.com/jorawar-singh/ optical_povm.

[51] A. Singh, Arvind, and K. Dorai, Witnessing nonclassical correlations via a single-shot experiment on an ensemble of spins using nuclear magnetic resonance, Phys. Rev. A 95, 062318 (2017). 